\title{
Relationship Between an Ischaemic J Wave Pattern and Ventricular Fibrillation in ST-Elevation Myocardial Infarction Patients
}

\section{Luyao Zhang* \\ Shujuan Dong* \\ Wenbo Zhao \\ Jingchao Li \\ Luqian Cui \\ Yongmei Han \\ Yingjie Chu $\mathbb{D}$}

Department of Cardiology, Henan Provincial People's Hospital, Zhengzhou, Henan Province, 450003, People's

Republic of China

*These authors contributed equally to this work
Correspondence: Yingjie Chu Email Chuyingjie59@163.com
Background: This study determined the ischaemic $\mathrm{J}$ wave pattern associated with ventricular fibrillation (VF).

Methods: A total of 262 patients diagnosed with ST-elevation myocardial infarction (STEMI) were recruited from October 2017 to September 2020. All data were collected and analysed, including baseline characteristics, electrocardiogram (ECG), coronary angiography (CAG), and examination outcomes.

Results: There were 193 STEMI patients with J wave elevation but without an ischaemic J wave (NJ group) and 69 patients with an ischaemic J wave; the latter were then subgrouped into early repolarization pattern (ERP; $n=62$ ) and Brugada pattern groups ( $B r P$ [anteroseptal ERP]; $n=7$ ). Univariate and multivariate logistic regression analyses were used to clarify high-risk factors and characteristics of ischaemic $\mathrm{J}$ waves. Multivariate logistic regression analysis revealed that an ischaemic $\mathrm{J}$ wave (odds ratio [OR], 9.708; 95\% CI, 2.570-36.664; $\mathrm{P}=0.01$ ) independently predicted VF. In the subgroup analysis, $\mathrm{BrP}(\mathrm{OR}, 31.214 ; 95 \% \mathrm{CI}, 3.949-246.742 ; \mathrm{P}=0.001)$, slur morphology of the ERP (OR, 8.15; 95\% CI, 1.563-42.558; $\mathrm{P}<0.05)$, and the number of leads with an ischaemic $\mathrm{J}$ wave $>3(\mathrm{OR}, 16.174 ; 95 \% \mathrm{CI}, 3.064-85.375 ; \mathrm{P}=0.001)$ were significantly associated with VF occurrence after adjusting for multiple variables.

Conclusion: An ischaemic J wave is an independent risk factor for VF in STEMI patients. BrP, slur morphology, and $>3$ leads with an ischaemic $\mathrm{J}$ wave could increase the incidence of VF.

Keywords: STEMI, ischaemic J wave, classification, predictor, ventricular fibrillation

\section{Introduction}

It has been more than 80 years since the $\mathrm{J}$ wave was first described by Shipley and Hallaran. ${ }^{1}$ The J wave appears as a "notch" or "slur" in the terminal part of the QRS complex. Both animal experiments ${ }^{2}$ and case-control studies ${ }^{3}$ have demonstrated that ischaemia induces and changes the electrocardiographic J wave. Therefore, we defined an ischaemic $\mathrm{J}$ wave as new onset or pre-existing. The $\mathrm{J}$ wave changes dynamically with the development of ischaemia with or without ST segment elevation and is induced by acute myocardial ischaemia, such as that associated with vasospastic angina $^{3}$ and acute myocardial infarction (AMI). ${ }^{4}$ It has been shown that an ischaemic $\mathrm{J}$ wave predicts malignant outcomes and long-term mortality. ${ }^{2,5}$

$\mathrm{J}$ wave syndrome (JWS) was first proposed by Yan et $\mathrm{al}^{6}$ in 2004. JWS is characterized by the appearance of a prominent electrocardiographic $\mathrm{J}$ wave with a risk of ventricular fibrillation (VF), including inherited Brugada syndrome (BrS), traditional repolarization syndrome (ERS), idiopathic ventricular fibrillation (IVF), 
and acquired arrhythmias linked to STEMI and hypothermia. Myojo et $\mathrm{al}^{7}$ reported that ischaemic $\mathrm{J}$ waves were observed during recurrent VF attacks in a patient with myocardial infarction, which implied that the appearance of $\mathrm{J}$ waves could be an important marker for lethal arrhythmias in acute ischaemia. Generally, J waves can be classified as inherited and acquired patterns. Inherited $J$ wave patterns, including the Brugada pattern (BrP [anteroseptal early repolarization pattern $\{E R P\}]$ ) and the ERP, have specific ECG characteristics, while acquired $\mathrm{J}$ waves, such as an ischaemic $\mathrm{J}$ wave, have a variety of patterns. ${ }^{8}$ Specifically, it has been reported that ischaemic $\mathrm{J}$ waves could also show the ECG characteristics of all inherited $\mathrm{J}$ wave patterns, including ERP and BrP. Therefore, we classified ischaemic $\mathrm{J}$ wave patterns into ERP and $\mathrm{BrP}$ types and studied the relationship between ischaemic $\mathrm{J}$ wave patterns and VF to provide clinical guidance for clinicians in managing these patients.

\section{Methods}

\section{Study Population}

This retrospective observational study consisted of 311 consecutive patients with STEMI who underwent primary percutaneous coronary intervention (pPCI) between October 2017 and September 2020 at the Henan Provincial People's Hospital (Zhengzhou, China). The diagnostic criteria ${ }^{9}$ for STEMI are in accordance with the universal definition of myocardial infarction (MI) as follows: an increase in troponin $>$ the 99th percentile of the upper reference limit; and new ST segment elevation at the J-point in 2 contiguous leads with cut-off points $>0.2 \mathrm{mV}$ in men or $>0.15 \mathrm{mV}$ in women in leads V2-V3 or $>0.1 \mathrm{~mm}$ in the other leads. The duration of symptoms related to ischaemia, such as chest pain, or other atypical symptoms, such as shortness of breath, nausea, vomiting, fatigue, palpitations, or syncope, was $<24 \mathrm{~h}$. Patients with a history of myocardial infarction, use of multiple-drug regimens, or anti-arrhythmic therapy were excluded. In addition, only patients receiving pPCI therapy were included in this study. After collecting and analysing all available ECGs, we excluded 2 patients with left bundle branch block (LBBB), 16 patients with right bundle branch block (RBBB), 22 patients who declined pPCI, and 4 patients with a duration of symptoms $>24$ h. Furthermore, 2 patients who had a prior AMI and 3 patients who lacked ECGs were also excluded. The remaining 262 subjects were included in this study.

\section{Data Collection}

According to the 2017 ESC guidelines for the management of AMI in patients presenting with ST-segment elevation, ${ }^{10}$ a standard 12-lead ECG was recorded for patients who complained of chest pain within $10 \mathrm{~min}$ after the initial medical contact. If the ECG was equivocal or did not support the diagnosis of MI, the ECGs were repeated and compared with previous recordings. In patients with a clinical suspicion of AMI and ST segment elevation, emergency reperfusion therapy was initiated as soon as possible. Following reperfusion, patients with STEMI were admitted to the coronary care unit (CCU) and provided continuous monitoring for at least $24 \mathrm{~h}$, and ECGs were remeasured at least once a day until discharge.

All data, including age, sex, smoking history, medical history, physical examination, all available ECGs, time from symptom onset to arrival at the hospital, coronary angiograms (CAGs), transthoracic echocardiograms, and blood sample tests, were recorded. The primary endpoint was the occurrence of VF recorded by ECG from the time of symptom onset. The content and procedures of this study were approved by the Henan Provincial People's Hospital Medical Ethics Committee (20171008).

\section{ECG Analysis}

All ECGs were scanned and magnified to $500 \%$ to measure the ECG parameters using Adobe Photoshop (version 20.0.0; Adobe Systems, Inc., San Jose, CA, USA), which was performed as previously reported. ${ }^{11}$ The ECGs were read by two independent cardiologists blinded to outcome status and clinical data. A third observer was consulted in the case of disagreement to make the final decision.

$\mathrm{J}$ wave was defined as a positive deflection immediately following the QRS complex of the surface ECG or buried in part inside the QRS as a notched (a positive J-deflection inscribed on the S wave) or slurred (a smooth transition from the QRS segment-to-the ST segment). ${ }^{12}$ $\mathrm{J}$ waves were considered present when the J-point was $\geq 0.1 \mathrm{mV}$ above the isoelectric line in $\geq 2$ contiguous leads of the 12-lead ECG with a QRS duration $<120 \mathrm{~ms}$. Ischaemic $\mathbf{J}$ waves were analysed from standard 12-lead ECGs, recorded at a paper speed of $50 \mathrm{~mm} / \mathrm{s}$ and calibration of $1 \mathrm{mV}$ per $10 \mathrm{~mm}$, based on the criteria of morphologic changes, voltage gain $(\geq 0.1 \mathrm{mV})$, and augmentation of $\mathrm{J}$ wave duration during ischaemia. The ECGs beginning with symptom onset were selected and analysed based on the following patterns: 
1. ERP: ${ }^{11}$ (1) an end QRS slur (Figure 1) or notch (Figure 2) on the downslope of a prominent $\mathrm{R}$ wave with and without ST-segment elevation; (2) the peak of the notch or $\mathrm{J}$ wave $\geq 0.1 \mathrm{mV}$ in $\geq 2$ contiguous leads of the 12-lead ECG; and (3) QRS duration (measured in leads in which a notch or slur is absent) $<120 \mathrm{~ms}$.

2. $\mathrm{BrP}$ (anteroseptal ERP): $:^{13}$ Type $1 \mathrm{BrP}$ (Figure 3) is characterized by ST-segment elevation $\geq 0.2 \mathrm{mV}$ in $\geq 2$ leads with ST segment descending elevation and T wave inversion. Types 2 and 3 were not specific for a $\mathrm{BrP}$, and only type 1 was recognized as a $\mathrm{BrP}$ in this study.

Patients with STEMI with ischaemic $\mathrm{J}$ waves were defined when the peak of a $\mathrm{J}$ wave $\geq 0.1 \mathrm{mV}$ and $\mathrm{J}$ wave $\geq 20 \mathrm{~ms}$ duration was observed. The ischaemic $\mathrm{J}$ wave was attenuated or had disappeared, and an MI did not develop after the patients underwent reperfusion therapy. In contrast, the patients without an ischaemic $\mathrm{J}$ wave were only shown to have $\mathrm{J}$ wave elevation. It should be noted that the ECGs of the patients before the onset of MI, as well as the early repolarization pattern, were not available.

\section{Statistical Analysis}

The continuous variables that were normally distributed are expressed as the means $\pm \mathrm{SD}$, and variables that had a skewed distribution are expressed as medians with 25th and 75th percentiles. Categorical variables are presented as counts and percentages. Continuous variables were compared using an unpaired $t$-test or the nonparametric Wilcoxon rank-sum test. Categorical variables were compared using the Pearson $\chi^{2}$ test or Fisher's exact test, as appropriate. Univariate analysis of the patient characteristics was compared between the groups with and without VF, and logistic regression analysis was performed to detect any independent significant predictors by multivariable adjustment (reported as odds ratios [ORs] with $95 \%$ confidence intervals [CIs]). The multivariate model for $\mathrm{VF}$ included age, sex, Killip class, potassium level, hyperlipidaemia, heart rate, and history of angina to clarify high-risk characteristics for ischaemic $\mathrm{J}$ waves. All analyses were performed using SPSS software (version 22.0; SPSS, Inc., Chicago, IL, USA). All tests were twotailed, and a $\mathrm{P}$ value $<0.05$ was considered statistically significant.

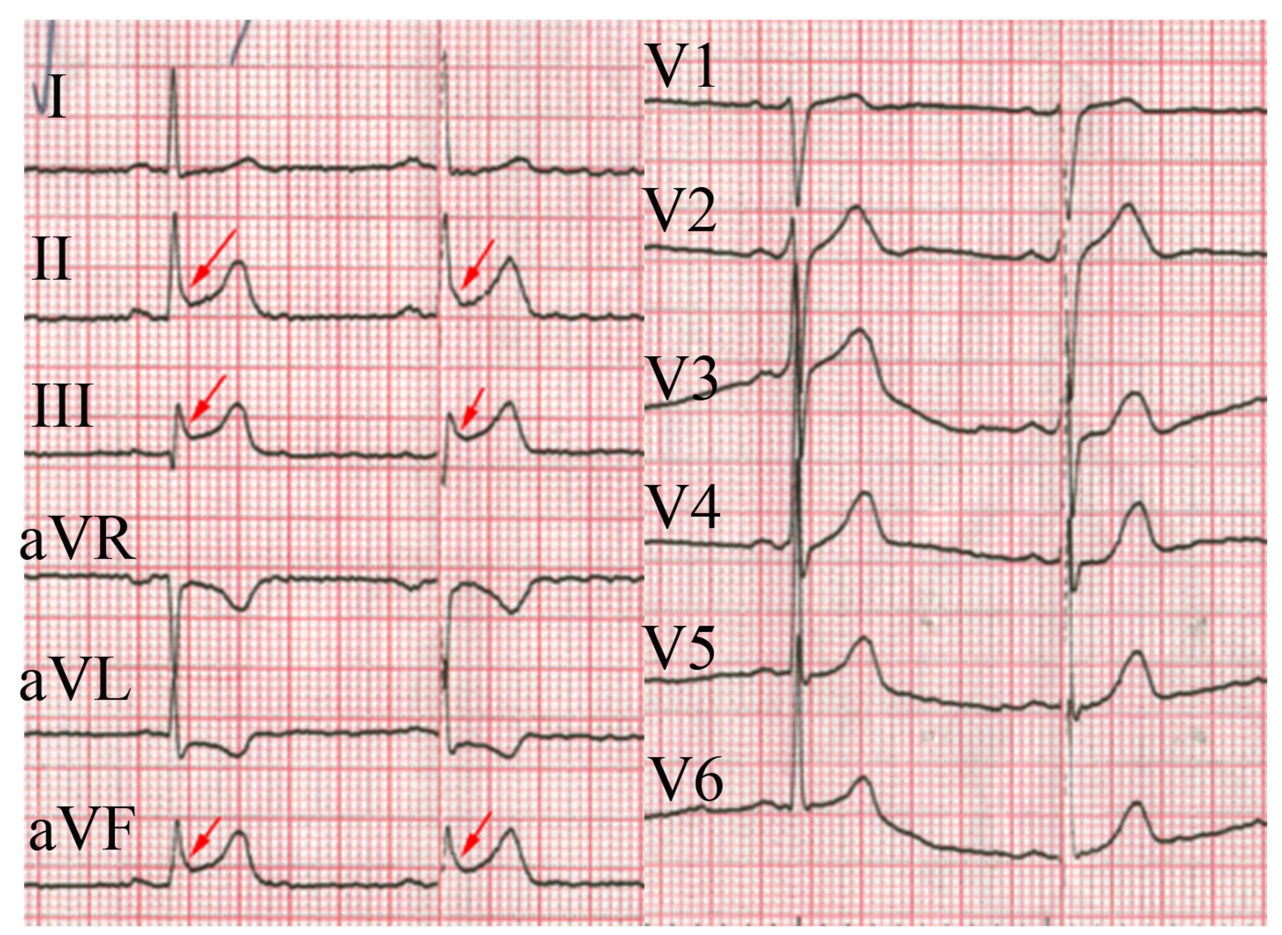

Figure I ECG of a 46-year-old man with chest pain for I hour at the time of admission. Leads II, III, and AVF show a slur-type ischaemic J wave (indicated by red arrows). CAG showed the culprit artery to be the LAD, which was consistent with the leads showing an ischaemic J wave. Twenty-seven minutes later, an ischaemic J wave in leads II and III and AVF disappeared, indicating that the J wave occurred in the very early phase and changed with the development of ischaemia. 


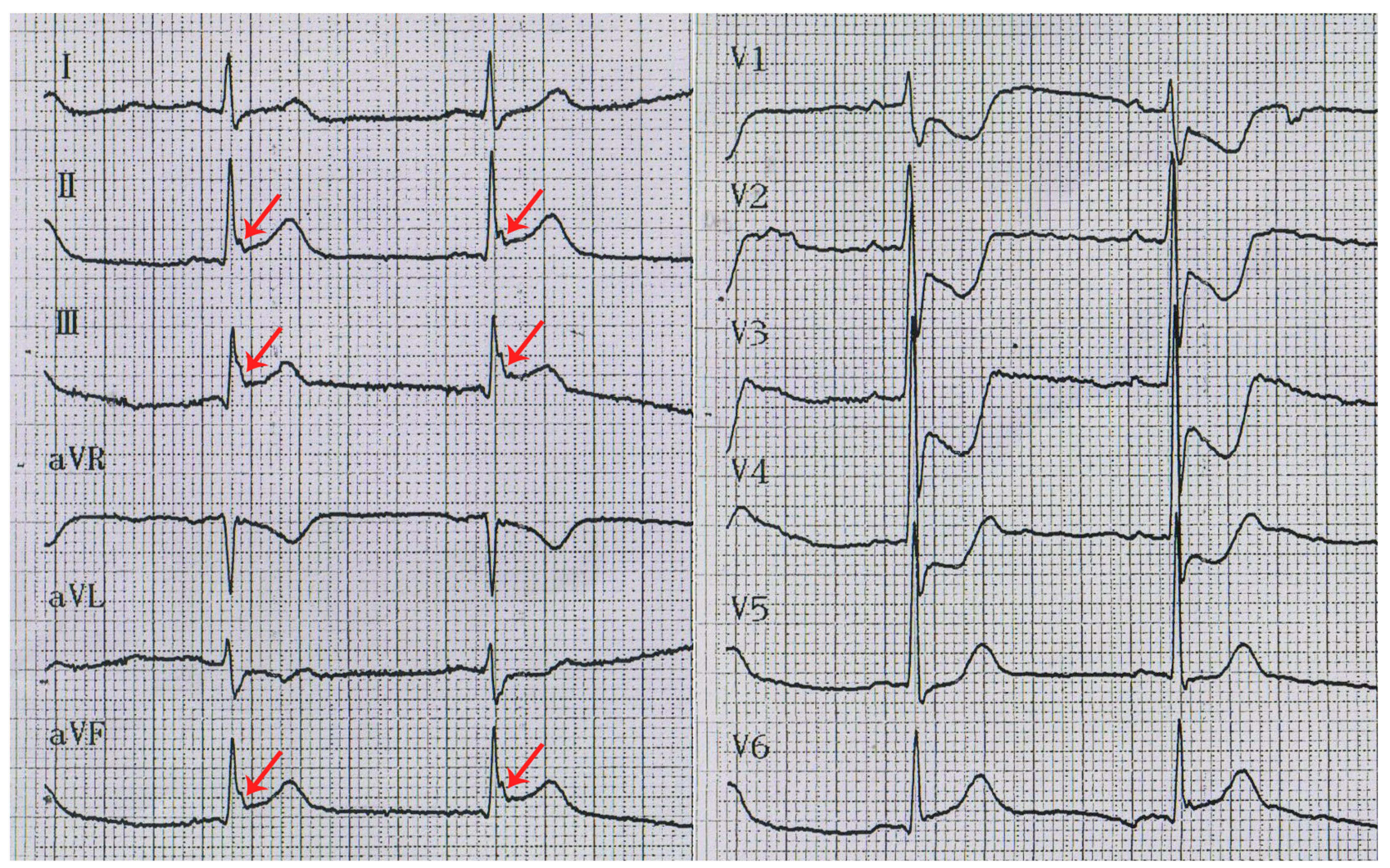

Figure 2 The ECG of a 55-year-old man presenting with chest pain for approximately I h. A notch J wave (indicated by red arrows) is present in leads II, III, AVF, and V6 with ST segment elevation consistent with the scope of blood supply to the RCA.

\section{Results}

\section{Demographic and Clinical Characteristics of AMI Patients}

Overall, ischaemic J waves were observed in 69 of 262 patients (26.3\%) with STEMI. As shown in Table 1, the baseline characteristics of the subjects were analysed based on ischaemic $\mathrm{J}$ wave patterns. The patients were separated into the following three groups: no $J$ wave (NJ) group ( $\mathrm{n}=193)$; ERP group $(\mathrm{n}=62)$; and $\mathrm{BrP}$ group $(\mathrm{n}=7)$. The majority of patients with STEMI were male $(81.3 \%)$, and $18.7 \%$ of the patients were female. There were no significant differences in sex, age, current smoking, diabetes, hyperlipidaemia, occurrence of angina in the past week, systolic blood pressure, elevated myocardium enzyme levels on admission, serum potassium level, creatine kinase level on admission, heart rate, or ischaemic $\mathrm{J}$ wave in the inferior leads. Interestingly, there was no significant difference in the percentage of ischaemic $\mathrm{J}$ waves in the inferior leads in the ERP and BrP groups (70.97\% vs $57.14 \%$; $\mathrm{P}=0.667$ ).

Compared with the NJ group, the ERP group had a shorter time from symptom onset to emergency room registration ( $4.3 \pm 3.4 \mathrm{~h}$ vs $6.6 \pm 4.9 \mathrm{~h}, \mathrm{P}=0.007)$. The Killip class IV rate in the BrP group was greater than that in the other two groups. In this study, $20(7.6 \%)$ patients had an episode of VF, and there were $12(4.6 \%)$ deaths from all causes after symptom onset. Among the 12 patients who died, VF was documented in 5 patients (41.7\%). Patients with an ischaemic $\mathrm{J}$ wave had a greater prevalence of $\mathrm{VF}$ $(\mathrm{P}<0.05)$ and all-cause deaths before hospital discharge than patients without an ischaemic $\mathrm{J}$ wave (Table 1), indicating that an ischaemic $\mathrm{J}$ wave might be a predictor of $\mathrm{VF}$ during AMI. Furthermore, 6 (85.71\%) most patients with VF were in the BrP group, while fewer patients were in the ERP group (11.29\%, $\mathrm{P}<0.001$; Table 1). In contrast, patients with an ERP had a lower mortality rate (4.84\%) than patients without an ischemic $\mathrm{J}$ wave $(2.59 \%$, $\mathrm{P}=0.407$; Table 1) compared to the $\mathrm{BrP}$ group (57.14\%), which was far greater than the $\mathrm{NJ}(\mathrm{P}<0.001)$ and ERP groups $(\mathrm{P}=0.001)$.

\section{Independent Predictors of VF}

A multivariate logistic regression analysis revealed that a history of angina (OR, 4.934; 95\% CI, 1.316-18.496; $\mathrm{P}<0.05$ ), Killip class IV (OR, 11.653; 95\% CI, 2.862- 


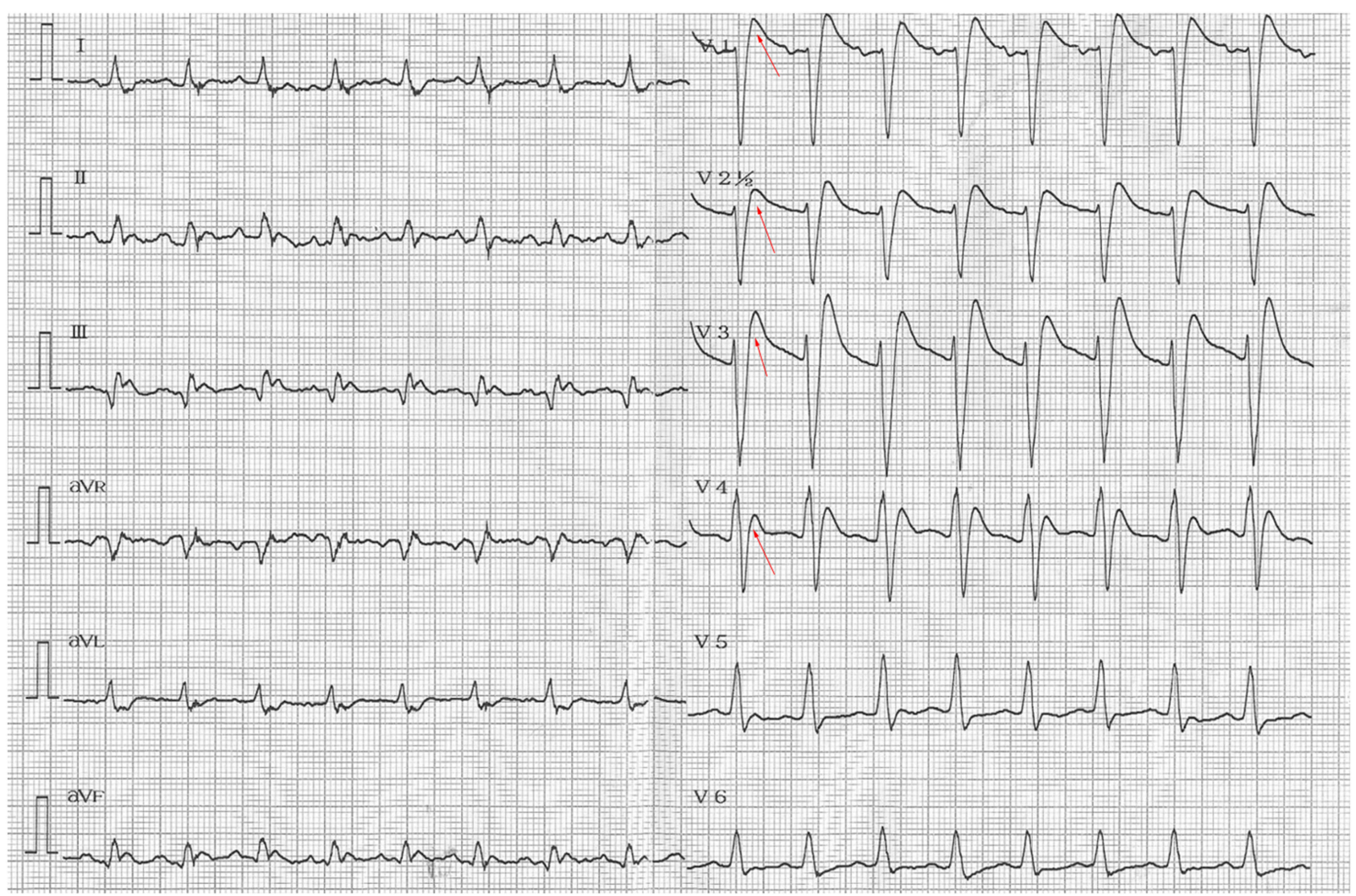

Figure $3 \mathrm{~A} \mathrm{BrP}$ in leads $\mathrm{VI}-\mathrm{V} 4$ and sudden cardiac death occurred in this patient. A BrP with ischaemic J wave is indicated by the red arrows.

47.438; $\mathrm{P}=0.001)$, potassium level in $\mathrm{mmol} / \mathrm{L}(\mathrm{OR}, 0.061$; 95\% CI, 0.013-0.293; $\mathrm{P}<0.001)$, heart rate (OR, 1.057; 95\% CI, 1.023-1.092; $\mathrm{P}=0.001)$, and the presence of an ischaemic $\mathrm{J}$ wave $(\mathrm{OR}, 9.708 ; 95 \% \mathrm{CI}, 2.570-36.664$; $\mathrm{P}=0.01$ ) were associated with $\mathrm{VF}$ after AMI onset (Table 2). Hyperlipidaemia was a protective factor based on univariate logistic regression analysis but not significant in multivariate logistic regression analysis, which might be affected by other variables. Furthermore, male sex, elderly, and creatine kinase levels on admission were not associated with VF (Table 2).

\section{Characteristics of an Ischaemic J Wave for Predicting VF}

Table 3 presents the multivariate-adjusted OR of VF associated with different ischaemic $\mathrm{J}$ wave characteristics. Patients with a BrP had a higher risk of VF (multivariate adjusted OR, 31.214; 95\% CI, 3.949-246.742; $\mathrm{P}=0.001$ ) than patients without an ischaemic $\mathrm{J}$ wave. Figures 3 and 4 show a representative case of a $\mathrm{BrP}$ in a patient with STEMI who developed VF during hospitalization.
Patients with an ERP did not have an elevated risk for VF (adjusted OR, 1.137; 95\% CI, 0.216-5.982, P>0.05). The results remained essentially the same based on multivariate analysis (Table 3). Among the 62 patients with an ERP, 20 (32.3\%) had the slur type, 20 (32.3\%) had the notch type at the end of the QRS complex, and $22(35.4 \%)$ patients had both slur and notch types. In the subgroup with an ERP, all of the slur (OR, 8.15; 95\% CI, 1.56342.558; $\mathrm{P}<0.05$ ), notch (OR, 1.458; 95\% CI, 0.233-9.114; $\mathrm{P}>0.05$ ) and both slur and notch types increased the occurrence of VF after multivariate adjustment; however, only the slur type $(\mathrm{P}<0.05)$ was statistically significant for predicting VF.

In addition, the number of leads with $\mathrm{J}$ waves were counted, and the results were as follows: 8 patients $(11.6 \%)$ with $>4$ leads; 37 patients $(53.6 \%)$ with 3 or 4 leads; and 24 patients $(34.8 \%)$ with $<3$ leads. There was no significant difference in the incidence of VF between the group with 1 or 2 leads and ischaemic $J$ waves and the group without an ischaemic J wave. Patients with 3 or 4 leads and an ischaemic $\mathrm{J}$ wave had a higher frequency of 
Table I Characteristics of STEMI Patients

\begin{tabular}{|c|c|c|c|c|c|c|}
\hline & $\begin{array}{l}\text { NJ Group } \\
(n=193)\end{array}$ & $\begin{array}{l}\text { ERP Group } \\
\quad(n=62)\end{array}$ & $\begin{array}{c}\text { BrP Group } \\
(n=7)\end{array}$ & $\begin{array}{c}\text { P value } \\
\text { (NJ vs ERP) }\end{array}$ & $\begin{array}{c}\text { P value } \\
(\mathrm{NJ} \text { vs } \mathrm{BrP})\end{array}$ & $\begin{array}{c}\text { P value } \\
(\text { ERP vs BrP) }\end{array}$ \\
\hline Male, \% & I57 (8I.3\%) & $50(80.6 \%)$ & $6(85.7 \%)$ & 0.902 & 1.000 & 1.000 \\
\hline Age, years & $57.6 \pm 12.8$ & $59.0 \pm 13.3$ & $64.9 \pm 13.0$ & 0.431 & 0.140 & 0.150 \\
\hline Current smoking, \% & II 3 (58.5\%) & 37 (59.7\%) & $2(28.6 \%)$ & 0.875 & 0.138 & 0.226 \\
\hline Hypertension & $87(45.1 \%)$ & 33 (53.2\%) & $3(42.9 \%)$ & 0.815 & 0.704 & 0.702 \\
\hline Diabetes & $52(26.9 \%)$ & $15(24.2 \%)$ & $2(28.6 \%)$ & 0.669 & 1.000 & 1.000 \\
\hline Hyperlipidaemia & 97 (50.3\%) & 23 (37.1\%) & I (I4.3\%) & 0.071 & 0.119 & 0.408 \\
\hline History of angina & 72 (37.3\%) & $20(32.3 \%)$ & $3(42.9 \%)$ & 0.472 & 1.000 & 0.679 \\
\hline Time from the symptom onset to the emergency room, $h$ & $6.0 \pm 5.9$ & $4.3 \pm 3.4$ & $6.6 \pm 4.9$ & 0.007 & 0.788 & 0.116 \\
\hline Systolic blood pressure, $\mathrm{mmHg}$ & $127.2 \pm 23.2$ & $120.8 \pm 23.4$ & $112.9 \pm 28.6$ & 0.061 & 0.112 & 0.407 \\
\hline Killip Class of IV on admission, $n$ (\%) & $15(7.77 \%)$ & $9(14.52 \%)$ & $4(57.14 \%)$ & 0.114 & 0.003 & 0.020 \\
\hline Elevated myocardium enzyme on admission, $\mathrm{n}(\%)$ & $103(53.4 \%)$ & $32(51.6 \%)$ & $3(42.9 \%)$ & 0.810 & 0.708 & 0.710 \\
\hline Potassium, $\mathrm{mmol} / \mathrm{L}$ & $3.99 \pm 0.50$ & $4.00 \pm 0.46$ & $3.87 \pm 0.61$ & 0.875 & 0.524 & 0.483 \\
\hline Creatine kinase levels on admission, umol/L & $72.50 \pm 29.23$ & $73.52 \pm 18.24$ & $78.57 \pm 25.11$ & 0.797 & 0.589 & 0.506 \\
\hline Heart Rate & $74.89 \pm 16.13$ & $73.05 \pm 18.23$ & $81.43 \pm 44.93$ & 0.451 & 0.714 & 0.642 \\
\hline \multicolumn{7}{|l|}{ Culprit artery } \\
\hline LM, LAD & 108 (54.40\%) & $16(25.81 \%)$ & $4(57.14 \%)$ & $<0.001$ & 1.000 & 0.181 \\
\hline LCX & 17 (8.8I\%) & $9(14.52 \%)$ & I (I4.29\%) & 0.196 & 0.489 & 1.000 \\
\hline $\mathrm{RCA}$ & 62 (42.49\%) & 37 (59.68\%) & $2(28.57 \%)$ & $<0.001$ & 1.000 & 0.226 \\
\hline Ischaemic J wave in inferior leads & $\longrightarrow$ & $44(70.97 \%)$ & $4(57.14 \%)$ & $\longrightarrow$ & $\longrightarrow$ & 0.667 \\
\hline Ventricular fibrillation & $7(3.63 \%)$ & 7 (11.29\%) & $6(85.71 \%)$ & 0.047 & $<0.001$ & $<0.001$ \\
\hline All cause death & $5(2.59 \%)$ & $3(4.84 \%)$ & 4 (57.14\%) & 0.407 & $<0.001$ & 0.001 \\
\hline
\end{tabular}

Abbreviations: NJ, nonischaemic J wave; ERP, early repolarization pattern; BrP, Brugada pattern, anteroseptal ERP.

Table 2 Univariate and Multivariate Logistic Regression Analyses of Ventricular Fibrillation Occurrence

\begin{tabular}{|c|c|c|c|c|}
\hline \multirow[t]{2}{*}{ Variables } & \multicolumn{2}{|l|}{ Univariate } & \multicolumn{2}{|l|}{ Multivariate $^{\dagger}$} \\
\hline & Odds Ratio (95\% Confidence Interval) & $P$ value & Odds Ratio (95\% Confidence Interval) & P value \\
\hline Age per year & $1.020(0.985-1.057)$ & 0.259 & 1.031 (0.980-1.084) & 0.237 \\
\hline Males & $2.169(0.486-9.675)$ & 0.310 & $3.594(0.590-21.902)$ & 0.165 \\
\hline History of angina & I.847 (0.739-4.6I4) & 0.189 & $4.934(1.316-18.496)$ & 0.018 \\
\hline Killip Class of IV & $8.610(3.205-23.129)$ & $<0.001$ & II.653 (2.862-47.438) & 0.001 \\
\hline Potassium per $\mathrm{mmol} / \mathrm{L}$ & $0.238(0.082-0.693)$ & 0.008 & $0.061(0.013-0.293)$ & $<0.001$ \\
\hline Hyperlipidaemia & $0.267(0.087-0.822)$ & 0.021 & $0.453(0.115-1.775)$ & 0.256 \\
\hline Ischaemic J wave & $5.760(2.196-15.113)$ & $<0.001$ & $9.708(2.570-36.664)$ & 0.001 \\
\hline Heart rate & $1.038(1.015-1.062)$ & 0.001 & $1.057(1.023-1.092)$ & 0.001 \\
\hline
\end{tabular}

Notes: ${ }^{\dagger}$ The variables that were included in the multivariate analyses were age, sex, history of angina, Killip class IV, potassium, hyperlipidaemia, ischaemic J wave and heart rate. Abbreviations: OR, odds ratio; $\mathrm{Cl}$, confidence interval; $\mathrm{NJ}$, nonischaemic J wave; ERP, early repolarization pattern; $\mathrm{BrP}$, Brugada pattern.

VF than patients without a $\mathrm{J}$ wave based on univariate (OR, 7.330; 95\% CI, 2.471-21.741; P<0.001) and multivariate logistic regression analyses $(\mathrm{OR}, 16.174 ; 95 \% \mathrm{CI}$, 3.064-85.375; $\mathrm{P}=0.001)$. Furthermore, the more leads affected by ischaemia, the higher the risk for VF.

\section{Discussion}

In this study, we showed that ischaemic $\mathrm{J}$ waves were found in $26.3 \%$ of patients with STEMI, and these patients had a greater prevalence of VF than patients without ischaemic J waves. Multivariate logistic regression analysis revealed that ischaemic $\mathrm{J}$ waves are an independent risk factor for VF in patients with STEMI. In the subgroup analysis, the BrP, slur morphology of the ERP, and the number of leads with an ischaemic $J$ wave $>3$ were significantly associated with VF occurrence. BrP and the number of leads with an ischaemic $\mathrm{J}$ wave $>3$ could increase the incidence of VF.

This study showed that there was no significant difference in the percentage of ischaemic $\mathrm{J}$ waves in the inferior 
Table 3 Univariate and Multivariate Logistic Regression Analyses of Ventricular Fibrillation

\begin{tabular}{|c|c|c|c|c|}
\hline \multirow[t]{2}{*}{ Variables } & \multicolumn{2}{|l|}{ Univariate } & \multicolumn{2}{|l|}{ Multivariate $^{\dagger}$} \\
\hline & Odds Ratio (95\% Confidence Interval) & $P$ value & Odds Ratio (95\% Confidence Interval) & $P$ value \\
\hline No ischaemic J wave & 1.00 & & 1.000 & \\
\hline \multicolumn{5}{|l|}{ Type of ischaemic J wave } \\
\hline ERP & $3.155(1.062-9.370)$ & 0.039 & $1.137(0.216-5.982)$ & 0.879 \\
\hline $\mathrm{BrP}$ & I56.857 (|6.573-|484.575) & $<0.001$ & 31.214 (3.949-246.742) & 0.001 \\
\hline \multicolumn{5}{|l|}{ Morphology of ERP } \\
\hline Notching & $1.598(0.334-7.646)$ & 0.557 & $1.458(0.233-9.114)$ & 0.687 \\
\hline Slurring & $3.596(1.050-12.321)$ & 0.042 & $8.155(1.563-42.558)$ & 0.013 \\
\hline Slurring and notching & $0.685(0.085-5.501)$ & 0.722 & $1.150(0.098-13.518)$ & 0.912 \\
\hline \multicolumn{5}{|c|}{ Number of leads with ischaemic J wave } \\
\hline $1-2$ & $2.416(0.472-12.359)$ & 0.290 & $3.490(0.504-24.163)$ & 0.206 \\
\hline $3-4$ & $7.330(2.47|-2| .74 I)$ & $<0.001$ & $16.174(3.064-85.375)$ & 0.001 \\
\hline$\geq 5$ & $15.943(3.161-80.407)$ & 0.001 & 36.045 (3.359-386.779) & 0.003 \\
\hline
\end{tabular}

Notes: ${ }^{\dagger}$ The variables that were included in the multivariate analyses were age, sex, Killip class of IV, potassium, hyperlipidaemia, heart rate, and history of angina. Abbreviations: OR, odds ratio; $\mathrm{Cl}$, confidence interval; NJ, nonischaemic J wave; ERP, early repolarization pattern; BrP, Brugada pattern, anteroseptal ERP.

leads in the ERP and BrP groups. However, the incidence of $\mathrm{VF}$ in the $\mathrm{BrP}$ group was greater than that in the ERP group. This finding might be because BrP increases the incidence of VF but does not affect other factors, such as the location of the $\mathrm{J}$ wave, which has been verified as a high-risk factor for VF. ${ }^{14,15}$ In addition, we found that the ERP group had a shorter time from symptom onset to emergency room registration than the $\mathrm{NJ}$ group. The reason for this finding is unclear but might be because the patients with an ischaemic $\mathrm{J}$ wave had a more severe STEMI that mimicked an ERP or was associated with the timing of ischaemic J waves, which may require further investigation.

The results of this study demonstrated the following: (1) an ischaemic J wave is an independent predictor for VF in the early phase of STEMI; (2) an ischaemic J wave can be divided into an ERP and a BrP; compared with an ERP, a BrP implies a higher risk of VF and occurs relatively later; (3) in terms of the morphology of the ERP, the slur type was significantly associated with the occurrence of VF compared with the notch type, which was inconsistent with findings in other studies; ${ }^{16-19}$ and (4) as the number of leads increased, the risk of VF also increased.

\section{Ischaemic J Wave as a Predictor of VF in Patients with STEMI}

As a marker of the very early phase of AMI, an ischaemic $\mathrm{J}$ wave appears in the first several hours and may disappear later or exist continuously, ${ }^{2,3}$ but the actual time window

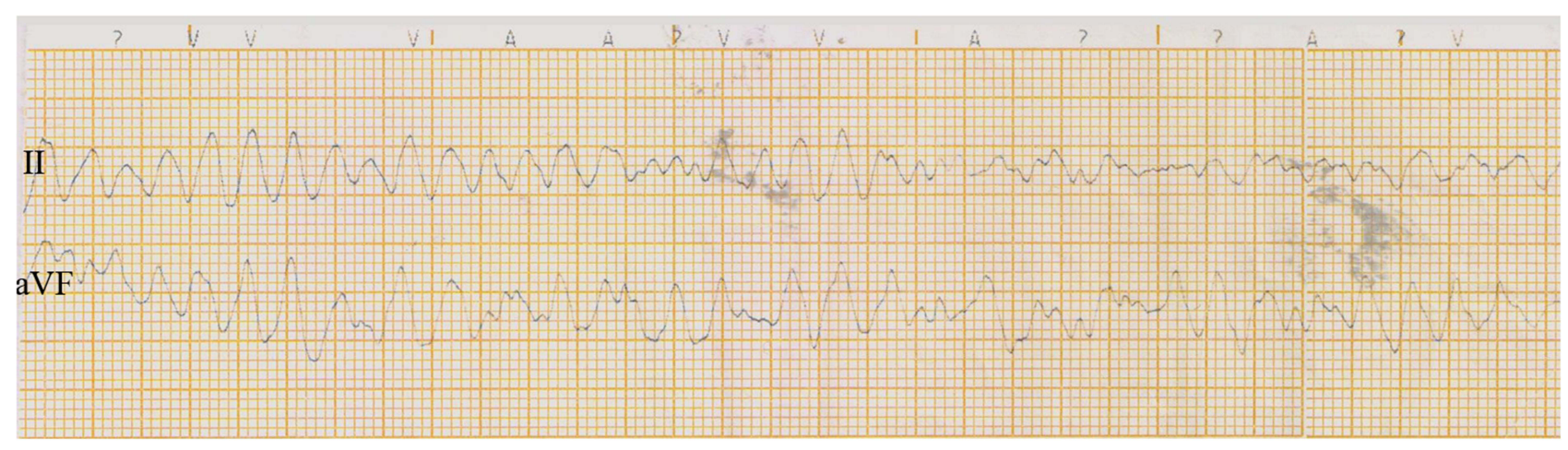

Figure 4 The records for the patient are shown in Figure 3. When he returned to the CCU after the procedure, ventricular arrhythmia was noted, and he was shocked immediately. He declined an ICD implant and requested discharge 7 days later. 
has not been established. In this study, the duration from symptom onset to the first ECG recorded in the ERP group was less than that in the $\mathrm{NJ}$ and $\mathrm{BrP}$ groups, indicating that although both an ERP and a BrP predicted VF in the early phase of a STEMI, ${ }^{2,20}$ the sensitivity of an ERP in predicting VF was less than that of a BrP. The percentage of patients with Killip class IV in the ERP and BrP groups was higher than that in the NJ group, which showed that a J wave might be related to haemodynamic instability but inconsistent with the findings reported by Ozcan et $\mathrm{al}^{20}$ and $\mathrm{Wu}$ et al. ${ }^{5}$ It is possible that the variable used for comparison between groups in these studies was the number of patients in Killip class $>1$ that did and did not undergo ERP.

After adjusting for variables that might have an influence on the endpoint in the present study by multivariable adjustment with multiple logistic regression analysis, the presence of an ischaemic $J$ wave was shown to be an independent risk factor for VF. A $\mathrm{J}$ wave on a surface ECG is located in the terminus of the QRS complex, which is a cut point between the ventricular depolarized (QRS complex) and repolarized (ST-T wave) components. It is still controversial whether a $\mathrm{J}$ wave is caused by late or early repolarization. The repolarization hypothesis ${ }^{21,22}$ maintains that repolarization dispersion in the early phases (phases 1 and 2) of the cardiac myocyte action potentials, which are created by a disproportionate amplification of the repolarized current in the epicardial myocardium due to a decrease in the inward sodium or calcium channel currents or an increase in the outward potassium currents mediated by the Ito, IK-ATP, and IK-Ach channels, are considered to be responsible for the inscription of the ECG $\mathrm{J}$ wave. With respect to the mechanism underlying VF mediated by a $\mathrm{J}$ wave, Yan et $\mathrm{al}^{23}$ demonstrated that acute transmural myocardial ischaemia results in a markedly heterogeneous loss of Ito-mediated epicardial AP domes across the ischaemic border, leading to Phase 2 re-entry. Phase 2 re-entry can in turn produce an R-on- $\mathrm{T}$ extrasystole that is capable of initiating VF.

Studies $^{4,24,25}$ supporting depolarization theory point out that slowing ventricular conduction allows the $\mathrm{J}$ wave to move out of the QRS complex resulting in an increase in J wave amplitude, characterized as tachycardiadependent accentuation. Nakayama et $\mathrm{al}^{4}$ reported that the $\mathrm{J}$-wave amplitude is not augmented at longer RR intervals but at shorter RR intervals, which demonstrated that the augmentation of the J-waves represents a decremental conduction that is inconsistent with traditional early repolarization theory. Similar findings have been reported by Sato et al. ${ }^{26}$ Di Diego et $\mathrm{al}^{27}$ showed that increased intracellular calcium activity contributes to the development of electrical inhomogeneity in the ventricle and the genesis of ventricular arrhythmias through triggered activity. Indeed, early repolarization (ER) or late depolarization could account for the mechanism underlying VF to some extent.

\section{Different Patterns for Predicting VF}

Azarov et $\mathrm{al}^{24}$ showed that prolongation of the activation time in ischaemic myocardium of domestic pigs is associated with J-wave generation and VF. Interestingly, Cipriani et $\mathrm{al}^{28}$ demonstrated that a specific ECG pattern, which is characterized by a "triangular QRS-ST-T waveform" and observed in 1.4\% of patients with STEMI, is associated with VF and cardiogenic shock. Moreover, other studies ${ }^{4,20,29}$ showed that both an ERP and BrP during AMI are correlated with an increased incidence of VF. In the current study, however, we found that only BrP predicted VF when comparing the subgroups of ischaemic $\mathrm{J}$ waves.

A number of studies ${ }^{4,5}$ have verified that an ERP predicts VF, especially in the setting of an MI, but an ERP in these studies was primary and diagnosed according to the ECG before an AMI. In the current study, the patients were grouped based on the presence or absence of an ischaemic $\mathrm{J}$ wave on admission ECG to determine whether a $\mathbf{J}$ wave is caused by acute myocardial ischaemia. Based on the results of multivariate logistic regression analyses, an ERP has been shown to be unrelated to VF. There are two reasons for this finding. First, a primary ERP is caused by the heterogeneous distribution of Ito in the epicardium and endocardium, while an ischaemic $\mathrm{J}$ wave is used for advanced repolarization in epicardial myocytes due to myocardial ischaemia. When the infarcted area undergoes reperfusion, an ischaemic $\mathrm{J}$ wave disappears, and the $\mathrm{VF}$ does not develop. Second, Demidova et $\mathrm{al}^{30}$ studied a large population with ER and pointed out that ER in patients with STEMI does not predict VF, which is consistent with our findings. Therefore, in this study, it can be concluded that $\mathrm{ER}$ is an independent predictor for VF, while the critical factor for predicting $\mathrm{VF}$ is a BrP. Large-scale multicentre clinical studies are needed to support this conclusion.

$\mathrm{BrP}$ is relatively rare and is more likely to induce VF. $^{31-33}$ Ferrando-Castagnetto ${ }^{25}$ reported a patient with a $\mathrm{BrP}$ as a dynamic electrocardiographic pattern during 
anterior AMI. Although studies involving the cellular basis of true BrS have been evolving, the physiologic mechanism underlying $\mathrm{BrP}$ in acute myocardial ischaemia is unclear. Di Diego et $\mathrm{al}^{27}$ analysed the potential physiologic similarities between ECG patterns observed in an experimental $\mathrm{BrS}$ model and an acute transmural no-flow ischaemia model using isolated canine right ventricle wedge preparations. The BrP model generated in preparations displaying a large epicardial Ito demonstrated that Ito modulates ECG manifestations of acute ischaemia similar to BrS, suggesting that both clinical entities are the result of a similar electrophysiologic substrate; however, the reason why this type of ischaemic $\mathrm{J}$ wave is more susceptible to VF is not clear.

\section{Characteristics of Ischaemic J Waves in Patients with STEMI and VF}

There are two ERP morphologies (notch and slur). Sato et $\mathrm{al}^{3}$ found that these two morphologies can be interconverted. Therefore, there might not be an absolute difference between malignant and benign patterns. Some studies ${ }^{16-18}$ have reported that the notch ERP is more prevalent in patients with STEMI and VF or sudden cardiac death, and a meta-analysis conducted by Zhang et $\mathrm{al}^{19}$ showed that patients with a notch $\mathrm{J}$ wave have a worse prognosis. Chen et $\mathrm{al}^{34}$ however, concluded that definite differences with respect to the prognostic significance between notch and slur ERPs have not been demonstrated. In contrast to the Chen et $\mathrm{al}^{34}$ findings, the presence of slur indicated a high risk for VF in our study. What needs to be emphasized is that the ERP in these studies was divided into slur and notch, and few studies involved patients with both notch and slur types in the same ECG. In the current study, unlike an inherited ERP, some patients presented two types of patterns, which could account for the above-described results.

In addition, the number of leads with an ischaemic $\mathrm{J}$ wave $>3$ had an increased OR for VF. Because acute myocardial ischaemia can occur in any part of the myocardial tissue, an acquired $\mathrm{J}$ wave could appear in each lead. Therefore, with exacerbation of the extent of ischaemia, the infarction area and involved myocardium gradually increased, and the number of leads with a $J$ wave increased accordingly. Furthermore, the number of leads with an acquired $\mathbf{J}$ wave is also affected by the density of Ito in the infarcted area. ${ }^{2}$

\section{Clinical Implications}

Our study demonstrated that an ischaemic $\mathrm{J}$ wave is an independent predictor of VF in patients with STMEI, and the risk of VF in patients with an ischaemic $J$ wave was 9-fold higher than that in patients without a $J$ wave. Specifically, more attention should be given to patients with BrP, the slur morphology of an ERP, and the number of leads with ischaemic $J$ waves $>3$ in the early phase of STEMI. In patients with an ischaemic $J$ wave, primary prevention of AMI is more important than in patients without an ischaemic $\mathrm{J}$ wave.

\section{Study Limitations}

First, this was a single-centre retrospective study, and the sample size was relatively small. Because of the low incidence of BrP in patients with STEMI, only 7 patients comprised the BrP group. Further investigations with a large sample size are required to confirm the current findings. Second, because all of the data were acquired by collecting the available information, it is possible that some patients with VF were missed. We monitored each patient in the same way; thus, this oversight occurred equally in the groups with and without $\mathrm{J}$ waves. Finally, we did not attempt to perform an electrophysiology study to elucidate the electrophysiologic characteristics of $\mathrm{J}$ waves in relation to the extent of scarring or cardiac function.

\section{Conclusion}

In this study, we showed that an ischaemic $\mathrm{J}$ wave is an independent risk factor for VF in patients with STEMI. Unlike ERP, another ischaemic $\mathrm{J}$ wave pattern $(\mathrm{BrP})$, as well as slur morphology and $>3$ leads with an ischaemic $\mathrm{J}$ wave, could increase the incidence of VF. These results may benefit clinicians in managing VF patients with ischaemic $J$ waves in the clinical setting.

\section{Data Sharing Statement}

The analysed datasets generated during the study are available from the corresponding author on reasonable request.

\section{Ethics Approval and Consent to Participate}

The content and procedures of this study were approved by the Henan Provincial People's Hospital Medical Ethics Committee (20171008). The study proceeded conforming to the ethical guidelines of the 1975 Declaration of Helsinki. Written consent from all participants was 
obtained for review of medical records and publication of clinical information.

\section{Funding}

There is no funding to report.

\section{Disclosure}

The authors report no conflicts of interest in this work.

\section{References}

1. Shipley RA, Hallaran WR. The four lead electrocardiogram in 200 normal men and women. Am Heart J. 1936;11:325-345. doi:10.1016/ S0002-8703(36)90417-9

2. Demidova MM, Martín-Yebra A, van der Pals J, et al. Transient and rapid QRS-widening associated with a J-wave pattern predicts impending ventricular fibrillation in experimental myocardial infarction. Heart Rhythm. 2014;11(7):1195-1201. doi:10.1016/j. hrthm.2014.03.048

3. Sato A, Tanabe Y, Chinushi M, et al. Analysis of J waves during myocardial ischaemia. Europace. 2012;14(5):715-723. doi:10.1093/ europace/eur323

4. Nakayama M, Sato M, Kitazawa H, et al. J-waves in patients with an acute ST-elevation myocardial infarction who underwent successful percutaneous coronary intervention: prevalence, pathogenesis, and clinical implication. Europace. 2013;15(1):109-115. doi:10.1093/ europace/eus 259

5. Wu CI, Chang SL, Lin CY, et al. Clinical significance of $\mathrm{J}$ wave in prediction of ventricular arrhythmia in patients with acute myocardial infarction. $J$ Cardiol. 2019;73(5):351-357. doi:10.1016/j. jjcc.2018.11.005

6. Yan GX, Yao QH, Wang DQ, Cui CC. J wave and J wave syndromes (in Chinese). Chin J Card Arrhythm. 2004;8:360-365.

7. Myojo T, Sato N, Matsuki M, Taniguchi O, Nakamura H, Hasebe N. An acute myocardial infarction case that survived an out-of-hospital cardiac arrest in which prominent ischemic $J$ waves were documented. Pacing Clin Electrophysiol. 2012;35(1):e27-e30. doi:10.1111/j.1540-8159.2010.02874.x

8. Antzelevitch C, Brugada P, Borggrefe M, et al. Brugada syndrome: report of the second consensus conference: endorsed by the Heart Rhythm Society and the European Heart Rhythm Association. Circulation. 2005;111(5):659-670. doi:10.1161/01. CIR.0000152479.54298.51

9. Thygesen K, Alpert JS, White HD; Joint ESC/ACCF/AHA/WHF Task Force for the Redefinition of Myocardial Infarction. Universal definition of myocardial infarction. J Am Coll Cardiol. 2007;50 (22):2173-2195. doi:10.1016/j.jacc.2007.09.011

10. Ibanez B, James S, Agewall S, et al. 2017 ESC guidelines for the management of acute myocardial infarction in patients presenting with ST-segment elevation: the Task Force for the management of acute myocardial infarction in patients presenting with ST-segment elevation of the European Society of Cardiology (ESC). Eur Heart J. 2018;39(2):119-177. doi:10.1093/eurheartj/ehx393

11. Akcay M, Yildırım U. Effect of dialysis and transplantation on myocardial repolarization parameters and $\mathrm{P}$-wave dispersion in chronic kidney disease. J Cardiol. 2021;37:1-8.

12. Macfarlane PW, Antzelevitch C, Haissaguerre M, et al. The early repolarization pattern: a consensus paper. $J$ Am Coll Cardiol. 2015;66 (4):470-477. doi:10.1016/j.jacc.2015.05.033

13. Pieroni M, Bellocci F, Crea F. Sudden cardiac arrest associated with early repolarization. $N$ Engl J Med. 2008;359(7):761-762.
14. Ralapanawa U, Kumarasiri PVR, Jayawickreme KP, et al. Epidemiology and risk factors of patients with types of acute coronary syndrome presenting to a tertiary care hospital in Sri Lanka. BMC Cardiovasc Disord. 2019;19(1):229. doi:10.1186/s12872-019$1217-\mathrm{x}$

15. Fumimoto T, Ueyama T, Shimizu A, et al. Inferior J waves in patients with vasospastic angina might be a risk factor for ventricular fibrillation. J Cardiol. 2017;70(3):271-277. doi:10.1016/j.jjcc.2016.12.003

16. Rudic B, Veltmann C, Kuntz E, et al. Early repolarization pattern is associated with ventricular fibrillation in patients with acute myocardial infarction. Heart Rhythm. 2012;9(8):1295-1300. doi:10.1016/j. hrthm.2012.03.006

17. Naruse Y, Tada H, Harimura Y, et al. Early repolarization is an independent predictor of occurrences of ventricular fibrillation in the very early phase of acute myocardial infarction. Circ Arrhythm Electrophysiol. 2012;5(3):506-513. doi:10.1161/CIRCEP.111.966952

18. Naruse Y, Nogami A, Harimura Y, et al. Difference in the clinical characteristics of ventricular fibrillation occurrence in the early phase of an acute myocardial infarction between patients with and without $\mathrm{J}$ waves. J Cardiovasc Electrophysiol. 2015;26(8):872-878. doi:10.1111/ jce. 12691

19. Zhang Z, Letsas KP, Yang Y, et al. Notch early repolarization pattern in inferior leads increases risk of ventricular tachyarrhythmias in patients with acute myocardial infarction: a meta-analysis. Sci Rep. 2015;5:15845. doi:10.1038/srep15845

20. Ozcan KS, Güngör B, Tatlısu MA, et al. Presence of early repolarization on admission electrocardiography is associated with long-term mortality and MACE in patients with STEMI undergoing primary percutaneous intervention. J Cardiol. 2014;64(3):164-170. doi:10.1016/j.jjcc.2013.12.007

21. Li GL, Yang L, Cui CC, Sun CF, Yan GX. J wave syndromes: a decade of progress. Chin Med J (Engl). 2015;128(7):969-975. doi:10.4103/0366-6999.154320

22. Antzelevitch C, Yan GX. J wave syndromes. Heart Rhythm. 2010;7 (4):549-558. doi:10.1016/j.hrthm.2009.12.006

23. Yan GX, Joshi A, Guo D, et al. Phase 2 reentry as a trigger to initiate ventricular fibrillation during early acute myocardial ischemia. Circulation. 2004;110(9):1036-1041. doi:10.1161/01.CIR.00001402 58.09964 .19

24. Azarov JE, Ovechkin AO, Vaykshnorayte MA, Demidova MM, Platonov PG. Prolongation of the activation time in ischemic myocardium is associated with J-wave generation in ECG and ventricular fibrillation. Sci Rep. 2019;9(1):12202. doi:10.1038/s41598-01948710-3

25. Ferrando-Castagnetto F, Garibaldi-Remuñan A, Vignolo G, RiccaMallada R, Baranchuk A. Brugada phenocopy as a dynamic electrocardiographic pattern during acute anterior myocardial infarction. Ann Noninvasive Electrocardiol. 2016;21(4):425-428. doi:10.1111/ anec. 12351

26. Sato A, Watanabe H, Sonoda K, et al. Augmentation of the J wave by rapid pacing in a patient with vasospastic angina. Int $J$ Cardiol. 2014;172(1):e111-e113. doi:10.1016/j.ijcard.2013.12.121

27. Di Diego JM, Antzelevitch C. High [Ca2+]o-induced electrical heterogeneity and extrasystolic activity in isolated canine ventricular epicardium. Phase 2 reentry. Circulation. 1994;89(4):1839-1850. doi:10.1161/01.CIR.89.4.1839

28. Cipriani A, D'Amico G, Brunello G, et al. The electrocardiographic "triangular QRS-ST-T waveform" pattern in patients with STsegment elevation myocardial infarction: incidence, pathophysiology and clinical implications. $J$ Electrocardiol. 2018;51(1):8-14. doi:10.1016/j.jelectrocard.2017.08.023

29. Alper AT, Tekkesin AI, Çinier G, Turkkan C, Baranchuk A. First description of a Brugada phenocopy in the inferior leads in the context of an acute inferior myocardial infarction. Europace. 2017;19(7):1219. doi:10.1093/europace/eux182 
30. Demidova MM, Carlson J, Erlinge D, Platonov PG. Early repolarization pattern on ECG recorded before the acute coronary event does not predict ventricular fibrillation during ST-elevation myocardial infarction. Heart Rhythm. 2020;17(4):629-636. doi:10.1016/j. hrthm.2019.11.011

31. Lippolis A, Buzzi PM, Romano IJ, De Concilio A, Gentile F. Unexpected reappearance of ST elevation in the anterior precordial leads shortly after an acute anterior myocardial infarction. $J$ Electrocardiol. 2019;52:75-78. doi:10.1016/j.jelectrocard.20 18.11.005

32. Eggebrecht H, Wieneke H, Erbel R. Image of the month. Brugada-like ECG pattern in a patient with isolated right ventricular infarction. Herz. 2009;34(4):327. doi:10.1007/s00059-009-3241-6
33. Pérez-Riera AR, Barbosa-Barros R, Daminello-Raimundo R, de Abreu LC, Baranchuk A. Unusual ST-segment elevation in the anterolateral precordial leads: ischemia, Brugada phenocopy, Brugada syndrome, all, or none? Circulation. 2017;136(20):1976-1978. doi:10.1161/CIRCULATIONAHA.117.031632

34. Chen Q, Zheng M, Liu G, et al. Early repolarization pattern predicts the increased risk of ventricular arrhythmias in patients with acute anterior ST-segment elevation myocardial infarction - a propensity analysis. Circ J. 2017;81(9):1346-1353. doi:10.1253/circj.CJ-161311
International Journal of General Medicine

\section{Publish your work in this journal}

The International Journal of General Medicine is an international, peer-reviewed open-access journal that focuses on general and internal medicine, pathogenesis, epidemiology, diagnosis, monitoring and treatment protocols. The journal is characterized by the rapid reporting of reviews, original research and clinical studies
Dovepress

across all disease areas. The manuscript management system is completely online and includes a very quick and fair peer-review system, which is all easy to use. Visit http://www.dovepress.com/ testimonials.php to read real quotes from published authors. 